\title{
Stochastic microstructure modeling and electrochemical simulation of lithium-ion cell anodes in 3D
}

\author{
Simon Hein ${ }^{\mathrm{a}, \mathrm{b}, \mathrm{c}, *}$, Julian Feinauer ${ }^{\mathrm{d}, \mathrm{e}}$, Daniel Westhoff ${ }^{\mathrm{d}}$, Ingo Manke ${ }^{\mathrm{f}}$, Volker Schmidt ${ }^{\mathrm{d}}$, Arnulf Latz ${ }^{\mathrm{a}, \mathrm{b}, \mathrm{c}}$ \\ ${ }^{a}$ German Aerospace Center (DLR), Institute of Engineering Thermodynamics, Pfaffenwaldring 38-40,70569 Stuttgart, Germany \\ ${ }^{b}$ Helmholtz Institute for Electrochemical Energy Storage (HIU), Helmholtzstraße 11, 89081 Ulm, Germany \\ ${ }^{c}$ Ulm University (UUlm), Institute of Electrochemistry, Albert-Einstein-Allee 47, 89081 Ulm, Germany \\ ${ }^{d}$ Ulm University (UUlm), Institute of Stochastics, Helmholtzstraße 18, 89081 Ulm, Germany \\ ${ }^{e}$ Deutsche ACCUmotive GmbH $\mathcal{E}$ Co. KG, Neue Straße 95, 73230 Kirchheim unter Teck, Germany \\ ${ }^{f}$ Helmholtz-Zentrum Berlin, Hahn-Meitner-Platz 1, 14109 Berlin, Germany
}

\begin{abstract}
Thermodynamically consistent transport theory is used to compare $3 \mathrm{D}$ images of real anode microstructures from lithium-ion batteries to virtual ones created by a parametric stochastic 3D microstructure model. Half-cell simulations in 3D with spatially resolved microstructures at different applied currents show that for low currents the deviations between various electrochemical quantities like current density or overpotential are negligibly small. For larger currents small differences become more pronounced. Qualitative and quantitative differences of these features are discussed with respect to the microstructure and it is shown that the real and virtual structures behave similar during electrochemical simulations. Extensions of the stochastic microstructure model, which overcome small differences in electrochemical behavior, are proposed.
\end{abstract}

Keywords: 3D microstructure modeling, lithium-ion battery, thermodynamically consistent transport theory, virtual materials testing, model validation

\section{Introduction}

Lithium-ion batteries play an important role in various sectors and applications. Their performance is highly influenced by the materials involved and the microstructure of the electrodes [1]. Thus, on the road to achieving high energy power densities, the optimization of the 3D microstructure of electrodes is an important task. Spatially localized features can have a strong impact on the overall battery performance and battery life expectation [2, 3]. Therefore, it is crucial to investigate cells not only regarding average structural quantities like e.g. mean porosity or tortuosity, but also to include the full 3D information. Techniques for structuring and directed creation of microstructures over several spatial magnitudes are getting employed more and more in lithium-battery research [4-6]. However, the problems of high costs for production and characterization, as well as low sensibility of imaging methods for certain materials

\footnotetext{
${ }^{*}$ Corresponding author:

Email address: Simon. Hein@DLR. de (Simon Hein)

URL: http://www.dlr.de/tt/en/ (Simon Hein)
} 
remain a problem. To overcome this, stochastic microstructure models for the 3D morphology of electrochemical materials can be used, see e.g. [7-11]. Once fitted to real tomographic image data, they serve as a tool to create virtual 3D microstructures on the computer by systematically varying the model parameters, which can be performed with much less effort than the production of those materials in the laboratory. Analyzing the electrochemical properties and performance of these structures on the computer using spatially resolved transport models yields information on which kind of microstructures enhance the performance of the corresponding materials. This procedure is called virtual materials testing. In the context of lithium-ion batteries, the resulting knowledge can then be taken into account when manufacturing real electrodes in the laboratory and thus potentially help to reduce the cost and workload of electrode optimization.

For anodes of lithium-ion cells, recently a stochastic 3D microstructure model has been developed, see [12]. The model has been fitted to tomographic image data, revealing a very good fit of morphological charcteristics, e.g., pore size distribution and local tortuosity. However, if we want to use this model for virtual materials testing, we need to make sure that it does not only match the morphological properties of real anode data, but also electrochemical characteristics, i.e., structures simulated by the aid of the calibrated model have to match the electrochemical properties of real data. Otherwise nothing could be concluded from the results concerning further virtual microstructures (created by varying model parameters). To show that the electrochemical properties of the simulated microstructures reflect the ones of real anode data is the topic of the present paper. This is done by using electrochemical simulations on tomographic image data of real battery anodes as well as on realizations of the calibrated stochastic model.

There are different approaches towards electrochemical modeling and simulation of lithium-ion batteries and the processes involved. The well-known porous electrode theory of Newman [13] and equivalent circuits models [14, 15] describe the microstructure of electrodes and separator as an averaged phase, which is defined by aggregate morphological parameters (e.g. tortuosity and mean porosities). However, here we want to include the whole 3D microstructural information to also account for the effect of local inhomogenities, which are neglected in 1D or pseudo 2D descriptions. Hence, electrochemical models which include the 3D resolved microstructure are of interest. Thus, in this paper, the thermodynamically consistent transport theory developed in [16, 17] is applied to both, realizations of the stochastic microstructure model and cut-outs of the tomographic image data with the same size. The quantities which influence possible degradation mechanisms [17, 18] were regarded with special attention. A good fit of various electrochemical properties between virtual and real image data is found, indicating that both would perform similar in a lithium-ion battery. Existing small differences between the virtual and real microstructures are addressed by proposed extensions to the stochastic microstructure model. We therefore claim that the stochastic 3D microstructure model can create microstructures which are behaving realistically regarding electrochemical performance. Thus, we conclude that the stochastic microstructure model is a valid tool for microstructure generation with the purpose of virtual materials testing.

The outline of the paper is as follows. In Sec. 2, the most important background knowledge is given, i.e., the stochastic microstructure model is shortly introduced in Sec. 2.1 and in Sec. 2.2 the 3D resolved electrochemical 
model is explained. The experimental background and technical details in this paper are summarized in Sec. 3 (where the experimental background is shortly summarized based on[19]). The results of our study are presented and discussed in Sec. 4 Conclusions and outlook can be found in Sec. 5 .

\section{Theory}

\subsection{Stochastic microstructure model}

In this section, we shortly recall some details of the stochastic 3D model that is used to generate the virtual anode microstructures. The model has been proposed in [12] and all parameters as well as further details can be found there. An overview of the generation of the virtual structures is given in Fig. 1 Generally, the model consists of three components: First, a random Laguerre tessellation, that decomposes the region of interest (ROI) into convex polytopes, is generated to determine the alignment of the particles as well as the size and coarse shape of each particle, see Fig. 1(a) Then, a (random) connectivity graph is realized to determine the connectivity between the particles and therewith connectivity of the whole particle system, see Fig. 1(b) Finally, random particles are placed into each polytope of the Laguerre tessellation, see Fig. 1(c) . Here, a random particle model based on Gaussian random fields on the sphere is used, which is capable of incorporating the boundary conditions given by the connectivity graph, i.e., particles touch each other where indicated by the graph. Finally, we delete the auxiliary tools (Fig. 1(d) and apply a morphological closing to mimic the effect of binder (Fig. 1(e)].

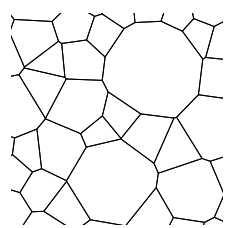

(a)

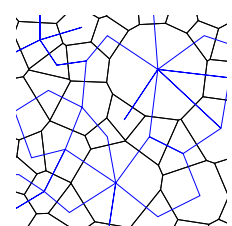

(b)

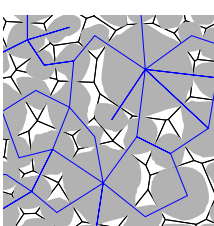

(c)

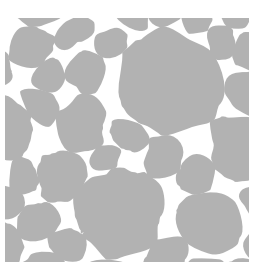

(d)

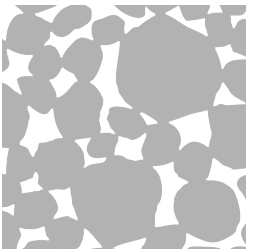

(e)

Figure 1: Schematic depiction of the stochastic model. (a) A random tessellation is generated, which roughly determines the particle shapes, sizes and locations. (b) A random graph describes how the particles connect. (c) The connected particles are then generated using Gaussian random fields on the sphere. (d) The auxilliary tools are deleted and the connected particle system is retained. (e) The particle system is morphologically smoothed to mimic the effect of binder on the contact region between particles. All pictures are taken from [12]. 


\subsection{Electrochemical model}

The lithium-ion battery considered in this paper is modeled electrochemically according to a thermodynamically consistent model [16, 17]. This model allows for a detailed 3D microstructure-resolved investigation. A short overview of the underlying theory is given in the following section.

\section{2.2.1. Thermodynamically consistent transport model}

The state of the lithium inside the electrolyte phase is given by the concentration $c_{\mathrm{El}}$ and the electrochemical potential $\varphi_{\mathrm{El}}$ and inside the solid phase by the concentration $c_{\mathrm{So}}$ and the electrical potential $\Phi_{\mathrm{So}}$. The mass balance equation and charge conservation equation are solved to obtain the time evolution of these quantities. The governing equations are listed in Eq. (1)

$$
\begin{array}{rlrl}
\partial_{t} c_{\mathrm{El}} & =-\boldsymbol{\nabla} \boldsymbol{N}_{\mathrm{El}} & \partial_{t} c_{\mathrm{So}} & =-\boldsymbol{\nabla} \boldsymbol{N}_{\mathrm{So}} \\
0 & =-\boldsymbol{\nabla} j_{\mathrm{El}} & 0 & =-\boldsymbol{\nabla} \boldsymbol{j}_{\mathrm{So}}
\end{array}
$$

with $N_{\mathrm{i}}$ as the lithium flux in the phase i:

$$
\begin{aligned}
& N_{\mathrm{El}}=-D_{\mathrm{El}} \nabla c_{\mathrm{El}}+\frac{t_{+}}{F} j_{\mathrm{El}} \\
& N_{\mathrm{So}}=-D_{\mathrm{So}} \nabla c_{\mathrm{So}}
\end{aligned}
$$

and $j_{\mathrm{i}}$ as the current density in the phase i:

$$
\begin{aligned}
& j_{\mathrm{El}}=-\kappa_{\mathrm{El}} \boldsymbol{\nabla} \varphi_{\mathrm{El}}-\kappa_{\mathrm{El}} \frac{1-t_{+}}{F}\left(\frac{\partial \mu_{\mathrm{El}}}{\partial c_{\mathrm{El}}}\right) \nabla c_{\mathrm{El}} \\
& j_{\mathrm{So}}=-\sigma_{\mathrm{So}} \nabla \Phi_{\mathrm{So}}
\end{aligned}
$$

The partial differential equations in the different phases are in contact at the corresponding interfaces. The particle and current fluxes are continuous. This is represented by the following equations (also called interface conditions):

$$
\begin{aligned}
\boldsymbol{j}_{\mathrm{El}} \cdot \boldsymbol{n}_{\mathrm{So}-\mathrm{El}}=i_{\mathrm{SoEl}} & \boldsymbol{j}_{\mathrm{So}} \cdot \boldsymbol{n}_{\mathrm{So}-\mathrm{El}}=i_{\mathrm{SoEl}} \\
\boldsymbol{N}_{\mathrm{El}} \cdot \boldsymbol{n}_{\mathrm{So}-\mathrm{El}}=\frac{i_{\mathrm{SoEl}}}{F} & \boldsymbol{N}_{\mathrm{So}} \cdot \boldsymbol{n}_{\mathrm{So}-\mathrm{El}}=\frac{i_{\mathrm{SoEl}}}{F}
\end{aligned}
$$

The normal of the interface area $\boldsymbol{n}_{\mathrm{So}-\mathrm{El}}$ is pointing from the solid (So) into the electrolyte (El). The interface current between solid and electrolyte is described by $i_{\text {SoEl }}$. Different reactions and types of interfaces can be expressed by using different expression for $i_{\text {SoEl }}$. In this paper, a symmetric Butler-Volmer (see Eq. (5)) is used for the interface current between the intercalation material (graphite) and the electrolyte. For more detailed information and explanations about the underlying theory the reader is referred to [16, 17]. The properties of the different materials (electrolyte, graphite, ...) are represented by their respective parameters (e.g.: lithium diffusion coefficient inside the anode $D_{\text {Anode }}$ ).

\subsubsection{Reactions}

In this paper, we investigate batteries in half-cell setup. As counterelectrode a lithium foil is used. We therefore assume lithium-intercalation into the graphite and lithium-deposition at the lithium-metal counterelectrode. 


\section{Intercalation}

The intercalation reaction from the electrolyte into graphite is described by

$$
\mathrm{Li}_{\mathrm{El}}^{+} \rightleftharpoons \mathrm{Li}_{\mathrm{So}}^{+}
$$

During the intercalation, lithium ions in the electrolyte shed their solvation shield, cross the interface between electrolyte and solid, and move into the solid phase. This reaction (R-Inter) is described with a symmetric Butler-Volmerequation,

$$
\begin{aligned}
i_{\mathrm{SoEl}}=2 & \cdot i_{\mathrm{SoEl}}^{00} \sqrt{c_{\mathrm{El}} c_{\mathrm{So}}\left(c_{\mathrm{So}}^{\max }-c_{\mathrm{So}}\right)} \\
& \cdot \sinh \left(\frac{F}{2 R T} \cdot \eta_{\mathrm{SoEl}}\right)
\end{aligned}
$$

The overpotential for the reaction is the difference between the electrochemical potentials of the lithium ions in the involved phases. A strict derivation leads to

$$
\eta_{\text {intercalation }}=\Phi_{\mathrm{So}}-U_{0}-\varphi_{\mathrm{El}}^{\mathrm{Li}^{+}}
$$

with the electrical potential $\Phi_{\text {So }}$ in the solid (graphite), the electrochemical potential $\varphi_{\mathrm{El}}^{\mathrm{Li}^{+}}$of the lithium ions in the

electrolyte and the open circuit potential (OCP) $U_{0}$ of lithium ions in graphite. [20]

\section{Lithium foil counterelectrode}

At the lithium-metal counterelectrode the lithium ions from the electrolyte are electrodeposited. This reaction can be written as

$$
\mathrm{Li}_{\mathrm{El}}^{+}+\mathrm{e}_{\mathrm{So}}^{-} \rightleftharpoons \mathrm{Li}_{\mathrm{Li}}^{0}
$$

The current flux at the the interface is expressed by a symmetric Butler-Volmer:

$$
i_{\mathrm{LiEl}}=2 \cdot i_{\mathrm{LiEl}}^{00} \cdot \sinh \left(\frac{F}{2 R T} \cdot \eta_{\mathrm{Li}}\right)
$$

with the overpotential

$$
\eta_{\mathrm{Li}}=\Phi_{\mathrm{Li}}-\varphi_{\mathrm{El}}^{\mathrm{Li}^{+}}
$$

\section{Experiments and Simulation}

\subsection{Measurement of microstructure}

In this paper two samples extracted from a large scale lithium-ion battery used in automotive applications have been considered. The cell was produced without electrolyte to ensure that the microstructure was not altered due to operation. To ensure that the samples are representative they have been taken from different positions and layers in the cell. The full procedure of the disassembly and preparation of samples is reported in [19]. 
The tomographic measurements of the microstructure were performed at the Synchrotron X-ray imaging facility BAMLine at BESSY (Berlin, Germany). The setup consists of a PCO4000 detector system with $4008 \times 2672$ pixels and an optical system (Optique Peter) with a CWO scintillator screen that was used to convert X-rays into visible light. An X-ray energy of $19 \mathrm{keV}$ was used. The voxel size is about $(0.44 \mu \mathrm{m})^{3}$. During the tomographic measurement, 2200 single radiographic projections were taken at equidistant angles over a range of $180^{\circ}$. A filtered back-projection algorithm was used for 3D data reconstruction. The reconstructed images have a resolution of $2097 \times 828 \times 119$ voxels. Each voxel has a grayscale value, which ranges from 0 to 255 .

Ten cut-outs with dimension $400 \times 400 \times 119$ are extracted from each of the two samples. These 20 cut-outs are the real microstructures used for the electrochemical simulations.

The virtual structures have been sampled directly from the model described in Sec. 2.1. To prevent edge effects, we sampled larger structures and took cut-outs with dimension $400 \times 400 \times 119$.

\subsection{Electrochemical simulations for validation}

The electrochemical simulations in this paper are conducted using the framework BEST (Battery and Electrochemistry Simulation Tool [21]), which is a finite volume implementation of the thermodynamic model derived in [16, 17]. Unfortunately, we obviously cannot compare our results to laboratory experiments, as the virtual structures only exist on the computer, however, the validity of the electrochemical model has been shown in [22]. The simulations are run on the high performance computing cluster JUSTUS, located at Ulm University.

\subsubsection{Microstructure}

The microstructures of the graphitic anode are based on tomographic image data (see Sec. 3.1) or created by the stochastic model (see Sec. 2.1) and are named "real" or "virtual" in the following, respectively. Real data or results therefore describe data and results obtained from electrochemical simulations using geometries based on tomographically reconstructed (real) microstructures. Statistical fluctuations in the real and virtual microstructures are incorporated by using 20 different cut-outs from the real data available and 20 realizations generated by the stochastic microstructure model. These microstructures consist of discrete volumes (voxels) with a side length of $0.44 \mu \mathrm{m}$ and have a thickness of 119 voxel or $52.36 \mu \mathrm{m}$ and width of 400 voxel $\times 400$ voxel or $176 \mu \mathrm{m} \times 176 \mu \mathrm{m}$. The direction from one current collector through the different layers towards the opposite current collector is named through direction. The directions perpendicular to through direction are called lateral directions (see also Fig. 2(a). The base size (lateral directions) is more than three times larger than the size of the sample in through direction and allows for a sufficient representation of possible lateral effects.

\subsubsection{Electrochemical simulations}

The battery setup in this paper is in half-cell configuration, which is operated with a constant current. In Fig. 2(a) a rendering of the battery geometry used for the electrochemical simulations based on one of the real cut-outs is shown. In our model, the through direction represents the full-cell thickness and all structural details are resolved. In 


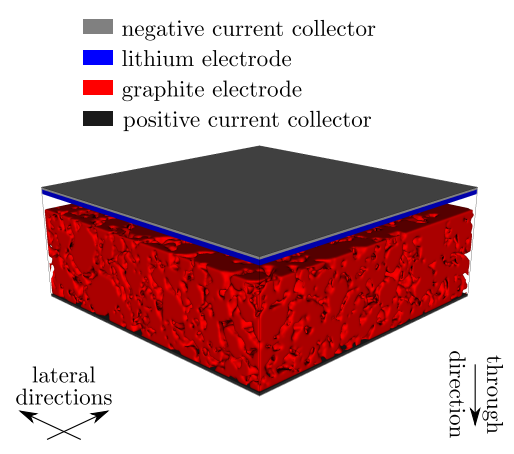

(a)

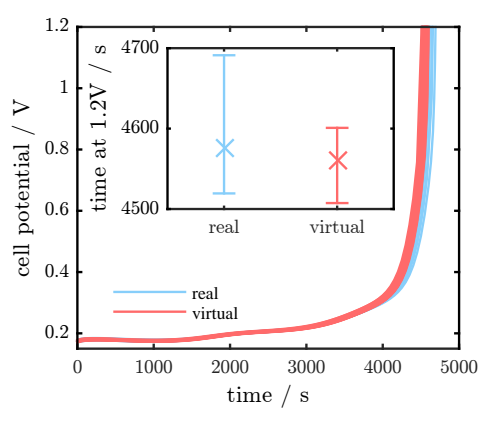

(b)

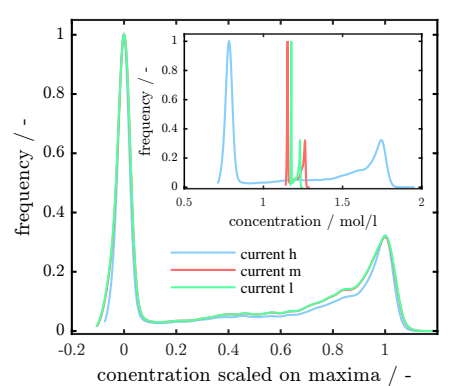

(c)

Figure 2: (a) The half-cell geometry used for the electrochemical simulations, here exemplified at the structure of a real cut-out. The different phases are (from top to bottom): Negative current collector, lithium electrode, separator/electrolyte (transparent), porous graphite and positive current collector. (b) (main) The cell potential of all full delithiation simulations show a good agreement between real and virtual microstructures. (inset) Comparison of the time when the cell potential reaches $1.2 \mathrm{~V}$, including the $5 \%$ - and $95 \%$-quantiles. (c) (inset) The distribution of the electrolyte concentration for the short current pulse at the three different applied currents. (main) The $x$-values of the concentration distribution are rescaled in such a way that the peak at high concentration is at the same position for all currents and vice versa for the low concentration peak. This shows that the three curves describe the same behavior and can be converted into each other by simple scaling.

lateral direction, insulating boundary conditions are assumed, i.e., neither lithium ions nor electrical current can leave the simulation domain in this direction. Periodic boundary conditions can not be used due to the fact that the real microstructures are cut-outs and the virtual microstructures are not created with periodicity in the different directions. Boundary conditions, which represent different operating conditions, are applied at the current collectors. While the negative current collector is set to a constant potential, a current flux is applied at the positive current collector. The total thickness of the battery in through direction is $66.44 \mu \mathrm{m}$ (151 voxel) for both types of microstructures. The current collectors have a thickness of 3 voxel. The lithium-foil electrode has a thickness of 6 voxel and the graphite electrode 119 voxel. The two electrodes are separated by a distance of 20 voxel. The separator is not resolved in this study, i.e., pure electrolyte is assumed here. When performing virtual materials testing, it is of course possible to represent the microstructure of the separator in 3D. However, only for the purpose of comparing the anode microstructures, this enhances complexity without any further benefit. The total number of discretization volumes of the geometries used for the electrochemical simulations is therefore 24160000 .

As first test the full delithiation of half-cells based on real and virtual microstructures was simulated electrochemically. Both types of battery microstructures are delithiated with the same constant current (current $\mathrm{m}$ in Tab. 1) starting at a state of charge of $95 \%$ until the cell potential reaches $1.2 \mathrm{~V}$. The resulting cell potentials of the full delithiation are shown in Fig. 2(b) The curves for real and virtual microstructures nicely coincide. A closer look at the point in time when the cell potential reaches $1.2 \mathrm{~V}$ illustrates that the real microstructures show slightly larger spreads in time compared to virtual microstructures. Nevertheless the cell potentials of real and virtual half-cells show the same type of behavior but also exhibit a deviation: at any given time during delithiation, the virtual structures always show 
a somewhat higher cell potential compared to the real ones. This is attributed to a slightly different overpotential caused by a difference in active area between real and virtual structures. This deviation is most pronounced for long simulations, but is also visible shortly after the simulation starts. On that account, a simulation duration shorter then a full delithiation or lithiation should suffice to allow for the investigation of electrochemical differences between the real and virtual microstructures. Hence, a current pulse duration with a duration of $10 \mathrm{~s}$ was chosen and all spatially resolved characteristics are investigated after $10 \mathrm{~s}$. Simulations have been performed with three different current densities (high, medium, low) to allow for different amounts of charge transferred during delithiation. They are listed with respect to the current collector area in Tab. 1. The current is applied at the positive current collector. The analysis of simulation results shows that the same microstructure behaves similar for different currents. A higher applied current leads to a more distinct effect for certain features. As an example, the distribution of electrolyte concentration at different applied currents for one real microstructure cut-out is shown in Fig. 2(c) A direct comparison of the histograms of the electrolyte concentration is not possible, therefore they are scaled in a way that the locations of the two maxima in the distribution are identical for the three currents. One can see that the shapes of the peaks are nearly identical. Also the features between the peaks exhibit a similar form. As these observations of similar trends and shapes were also made for other quantities, further discussions and comparisons of real and virtual microstructures are only shown for the highest current (current h). As stated before, for some quantities a stronger separation between interesting features occurs for the highest current.

\subsubsection{Parameters}

The half-cell simulation uses the porous graphite electrode with a flat lithium counter-electrode. The separator is represented by an effective porous medium between the two electrodes. In Tab. 2 the material parameters needed for the electrochemical simulations for the various phases are listed. We chose the parameters in accordance with common literature. Note that in the present study, we focus on morphological and geometrical material parameters rather than chemical and physical ones, thus, it is sufficient to chose quite arbitrary, but realistic parameters to compare real and virtual morphologies. As electrolyte $\mathrm{LiPF}_{6}$ in PC/EC/DMC was used. All simulations are conducted isothermally at the same temperature $(T=298 \mathrm{~K})$, hence temperature effects are neglected. This assumption is valid since a previous study [17] showed that the main heat production is due to the irreversible Joule heat at the interface between active material and electrolyte, and thus only depends on the active surface area, which only slightly differs between real and virtual structures.

\section{Results}

Throughout this section, blue color indicates results for real structures, whereas red color is used for virtual structures. Solid lines show the course of the mean value of a quantity and the region between the $5 \%$ and $95 \%$ quantile is shaded in the respective color. All figures showing spatially resolved characteristics are based on the data at the end of the discharge pulse $(t=10 \mathrm{~s})$. 
The cell potential of a battery during operation is one of the quantities used to compare different cells. The average cell potentials for the real and virtual cut-outs are shown in Fig. 3 . Variations of the microstructures for different cut-outs lead to slightly different active surface areas and solid volume fractions. This results in a scattering of the cell potentials, which is more pronounced for the real microstructures. A larger surface area leads to a lower interface overpotential and the cellpotential is thus lower. Since the current is applied at $t=0 \mathrm{~s}$, the cell potentials already differ. With zero applied current the cell potentials would show the same cell potential, namely the value of the open-circuit-potential $U_{0}\left(c_{\text {Initial }}\right)$ at initial state of charge. The virtual cut-outs therefore exhibit a overall lower cell potential as compared to the real ones. Although the cellpotential of the real and virtual cut-outs overlap withing the quantile regions, a fine-tuning of the stochastic microstructure model is possible. In Tab. 3 the statistics for the

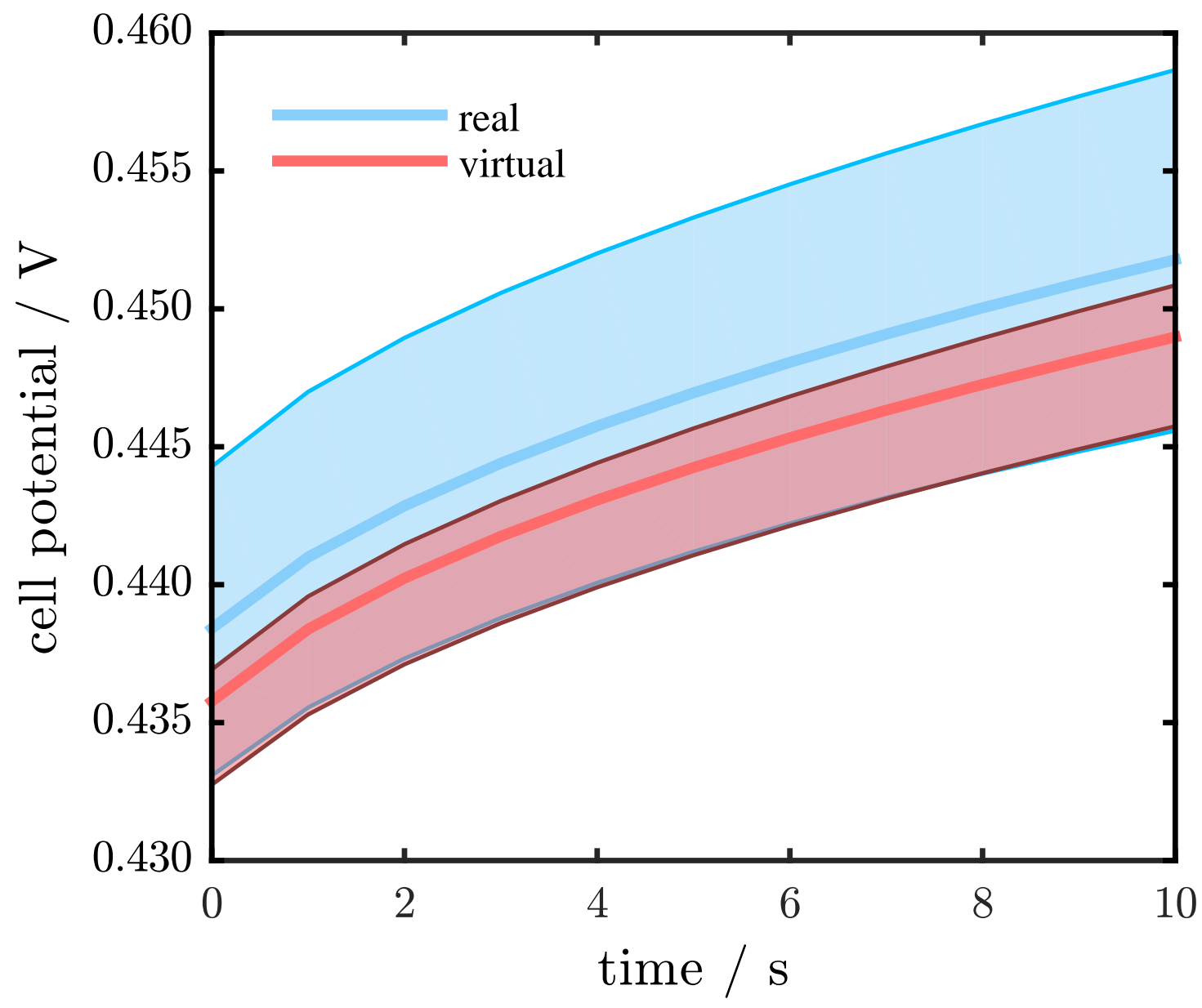

Figure 3: The average cell potential for real and virtual structures during the first $10 \mathrm{~s}$. The region between the $5 \%$ and $95 \%$-quantile is shown as shaded area.

surface area and the solid volume of the real and virtual microstructures are listed. The surface area of the voxelized 
structures (see Tab. 3) shows a different trend compared to the surface area of the smoothed (virtual) structures (see Fig. 1(e) and [12]). These differences are a result of the voxelization process, which converts smooth geometries into structures consisting of cubic voxels. Nevertheless, the deviations between mean cellpotential of the real and virtual microstructures are less than $1 \%$ or $5 \mathrm{mV}$ and thus the microstructures show a very good agreement. While the cut-outs of the real microstructures exhibit a spread between minimal and maximal surface area of $16 \%$, the cell potentials vary by less then $3 \%$. A similar trend is found for the virtual structures. Thus, we conclude that real and virtual microstructures are not discriminable from overall cell performance data.

Additionally the effective diffusion coefficient and the effective conductivity in through-direction were calculated using the software GeoDict [23]. The resulting values are listed in Tab. 4. The effective diffusion coefficients differ significiantly between real and simulated structures (considering the standard deviation). This is attributed to the inhomogeneous spatial distribution of the solid volume fraction. In Fig. 4 the spatial distribution of the solid volume fraction (SVF) is shown for the real and virtual microstructures. The average SVF for the real microstructures exhibits an increase at both external interfaces in through direction. At the region close to the current collector and close to the separator the solid phase is more dense. This is not resembled by the stochastic microstructure model. By removing 10 voxels on each side in through direction, the more dense region is removed from the real microstructures (see Fig. 4). Now the difference in effective diffusion coefficients is less strong (see Tab. 4). The microstructures show a similar effective conductivity and the values for the virtual microstructures are within the error margin of the ones of the real microstructures. The influence of the more dense regions of solid volume fraction on electrochemical quantities is discussed in the following sections. As a next step, we examine several intrinsic quantities more closely. In the following all quantities are taken at the end of the delithiation simulation at $t=10 \mathrm{~s}$.

\subsection{Electrolyte concentration}

The first quantity of interest is the electrolyte concentration. The temporal evolution of this quantity during the electrochemical simulations is highly influenced by the $3 \mathrm{D}$ microstructure and is therefore sensitive to possible deviations between the real and virtual microstructures. The distribution of the electrolyte concentration at the end of the discharge pulse (see Fig. 5(a) is considered separately for the pore space of the electrode and the separator (inset). The small peak at $1.2 \mathrm{~mol} / 1$ indicates volumes of the pore space in the electrode, which are not connected to the main pore network. In real microstructure these unconnected regions would not be filled with electrolyte. In the simulations these volumes were filled with electrolyte, but they stay electrochemically inactive. Hence these "unconnected pore volumes" appear as a small peak at the initial concentration. The virtual structures exhibit more unconnected pore space than the real microstructures. This might occur due to the connection rule of particles in the stochastic microstructure model. The concentration maxima in the virtual structures are at slightly lower positions than for the real ones. This is also visible in the 3D-spatial distribution for two selected cut-outs (see Fig. 5(c)). The virtual structures show an overall smaller spread between minimal and maximal concentrations as well. Therefore the electrolyte concentration distribution of the virtual cut-outs inside the separator (inset in Fig. 5(a) is shifted to a 


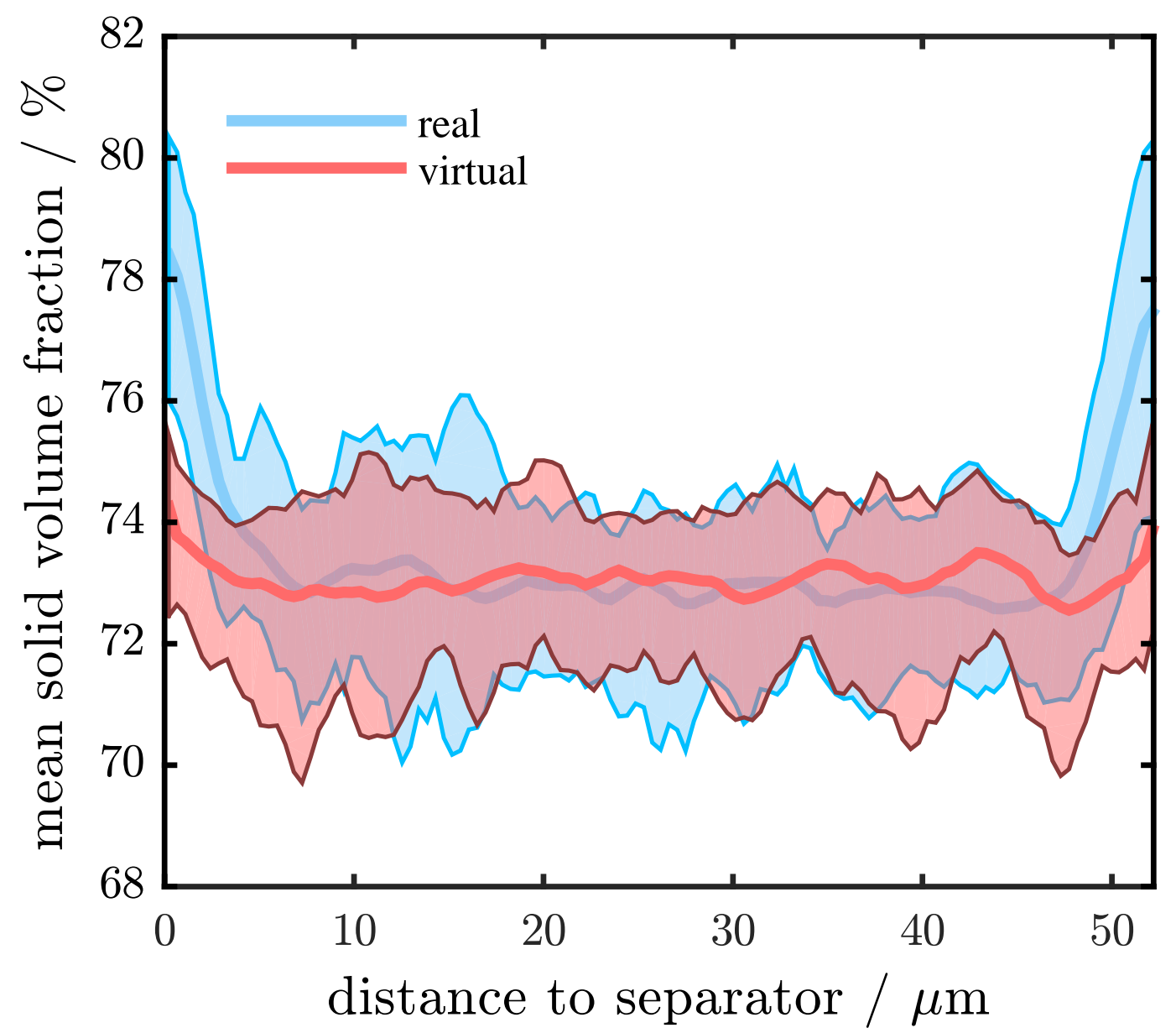

Figure 4: The average solid volume fraction along the through direction for the real and virtual structures. The spatial distribution for the real cut-outs shows a region of more dense material close to the separator and close to the current collector. This property is not fully present in the virtual structures.

higher position than for the real ones. This effect is attributed to the slightly larger total electrolyte volume (see Tab. 3 ) and the accordingly minor spatial concentration gradients.

In Fig. 5(b) the average electrolyte concentration as a function of the distance to the separator is displayed. The shapes of the concentration functions for the real and virtual structures are nearly identical. Close to the current collector the concentration is somewhat higher for the real structures compared to the virtual structures. The nonlinear gradient of the concentration is due to the superposition of transport and delithiation. Since there are no lithium ion sources in the separator, the lithium diffusion and migration lead to a linear concentration gradient. The small difference between the concentration functions for real and virtual structures is probably due to the small differences in electrolyte volume (see Tab. 3). The shape of the spatial distribution of the mean electrolyte concentration is quite 


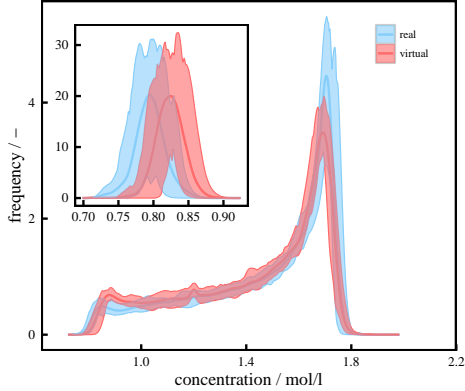

(a)

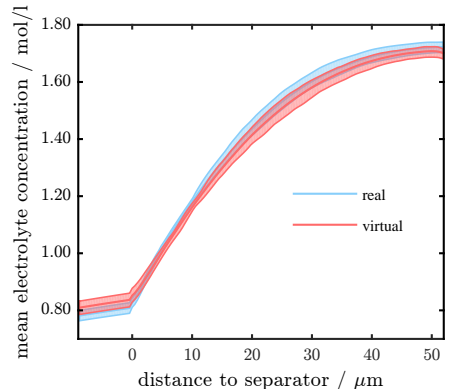

(b)

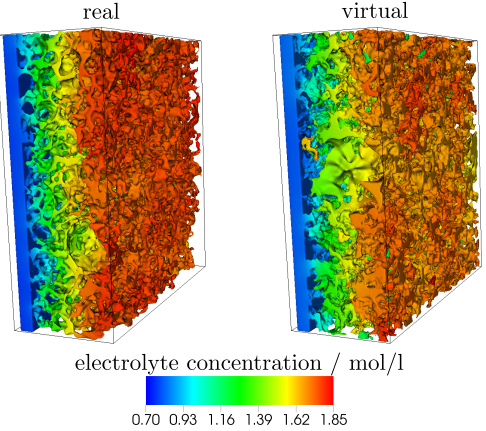

(c)

Figure 5: (a) The distribution of the electrolyte concentration for the electrode pore space and the separator (inset). The density is normed to unity area. The small peak at the initial concentration indicates unconnected pore volume. (b) The mean lithium concentration in the electrolyte as a function of the distance to the separator. The shape and height are nearly identical and both curves overlap in the region between the $5 \%$ and $95 \%$-quantiles. (c) Spatial distribution of electrolyte concentration for two cut-outs: real (left) and virtual (right). Both have the same color scale (shown below). Larger particles can be seen in both structures. Also both cut-outs show electrolyte pores, which are less connected to the main pore space: (virtual) Orange part close to the blue and (real) dark red at the upper corner.

similar to what is usually obtained as a result of porous electrode theory [17, 24]. One of the advantages of the 3D simulation is that local effects are easily resolved and no special assumptions are needed. In Fig. 5(c) the spatial distribution of electrolyte concentration is shown for two cut-outs of real and virtual microstructures. In both cut-outs some less-than-average connected pores can be seen: The small orange/yellow pore close to the separator and the dark red in the lower corner for the virtual structure and the dark red at the upper current collector interface for the real structure. The concentration profile of the real structure shows higher concentration (more dark red) compared to the virtual microstructure. This is in agreement with the slight difference in the position of the concentration peak in Fig. 5(a)

\subsection{Current density}

The current density is a quantity, which influences the degradation inside a battery. Lithium plating, SEI growth and electrolyte decomposition are some of the reactions, which are highly influenced by the local current flow [18]. High currents increase the local temperature [17] and hence lead to local decomposition of SEI, or, in the worst case, to start exothermic reactions which lead to thermal runaway.

The current density in the electrolyte and solid phase is defined in Eq. (3). At the interfaces between the solid phase and the electrolyte, the current density is continuous and given by the interface condition

$$
\boldsymbol{j}_{\mathrm{i}} \cdot \boldsymbol{n}=i_{\mathrm{So}-\mathrm{El}}
$$

with the interface current $i_{\mathrm{So}-\mathrm{El}}$ as defined by Eq. (5). The charge carriers inside the electrolyte are lithium ions and electrons inside the solid phase. 


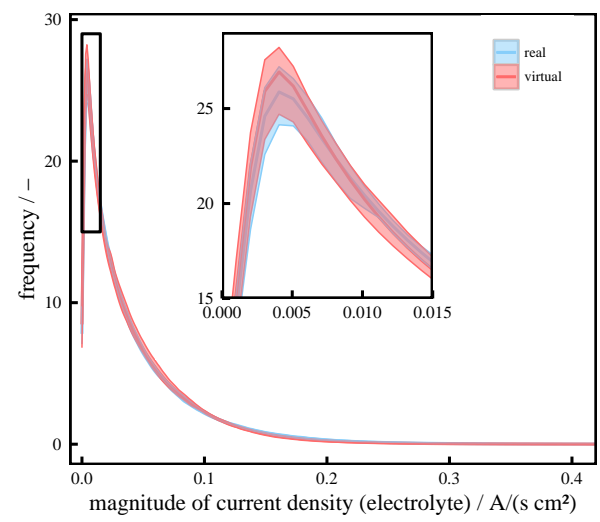

(a)

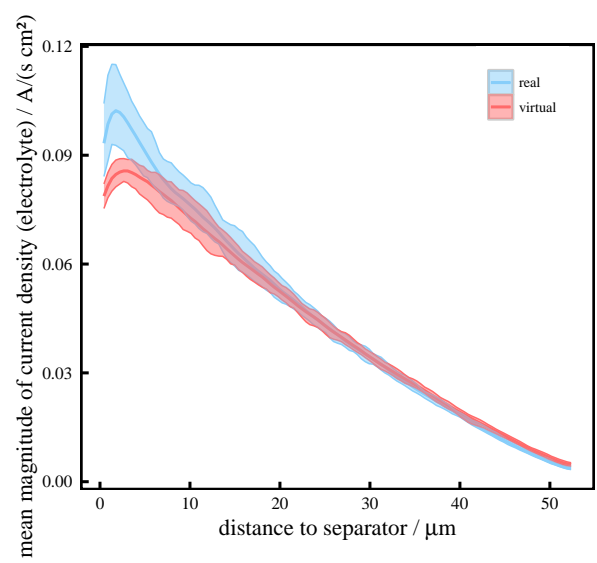

(c)

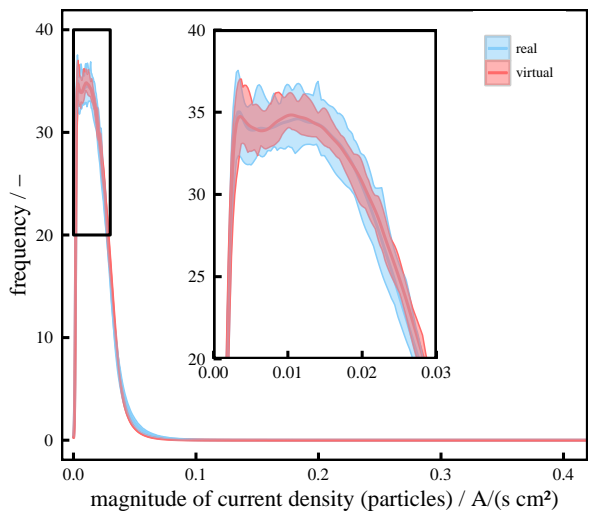

(b)

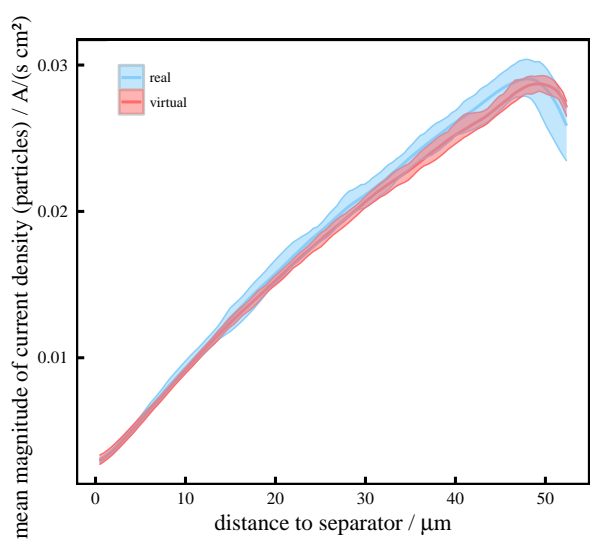

(d)

Figure 6: Histogram of the current density inside the electrolyte (a) and the solid phase (b) for the high applied current after 10 s. While lithium ions are the charge carrier in the electrolyte, electrons fulfill this role in the solid phase. The distributions of the current densities in the real and virtual structures show a nearly identical behavior. The spatial distributions of the average current density inside the electrolyte (c) and inside the solid phase (d) for high applied current after $10 \mathrm{~s}$. The spatial distribution of the average current densities of real and virtual structures differ inside the electrolyte close to the separator and inside the solid phase close to the current collector.

\subsubsection{Electrolyte phase}

The current densities in the electrolyte phase of the real and virtual microstructures show a nearly identical behavior (see Fig. 6(a) . The virtual structures contain slightly more regions with smaller current densities. For higher local current densities, which could be critical for SEI decomposition, no differences can be seen between the two microstructure sources. This is one of the indications that the virtually generated microstructures are electrochemically very similar to the real structures.

The spatial distribution of the average current density magnitude is shown in Fig. 6(c) The current density increases from the current collector towards the separator, since the charge transport changes from solid to electrolyte 
phase. The largest deviation between current densities of the virtual and real structures is observed close to the separator. The real microstructures display a current density, which is roughly $10 \%$ higher than the values for the virtual structures close to the separator. We attribute this difference to the increase in the solid volume fraction (SVF) close to the separator for the real microstructures, which is not resembled by the model and leads to a decrease of electrolyte volume. This, in turn, leads to an increase in the current density, since the same amount of current needs to flow through a smaller volume.

\subsubsection{Solid phase}

In Fig. 6(b) the distribution of the current density in the solid is shown. The frequencies of the current densities in real and virtual microstructures both show a broad peak for small values of the current density. This peak has two smaller peaks as features, which are shown as inset, and are present in both, virtual and real structures. Similar to the spatial distribution of the current density in the electrolyte, the current density inside the solid phase of the electrode shows a deviation close to one of the electrode interfaces (see Fig.6(d)). For the solid phase, the slope of the current density is reverted compared to the electrolyte phase, since closer to the separator more and more charge gets transported through the electrolyte phase. Close to the current collector two features are visible: the average current density in the solid phase increases for some distance (roughly $5 \mu \mathrm{m}$ ). In the same region, the values for real structures are smaller than for the virtual ones. As soon as the current density starts to decline, the values of the real structures are larger. Both features are accounted on the spatial variance of the solid volume fraction (see Fig. 4p: The increase in current density is a result of the decreasing solid volume fraction in this spatial range, because the same current across a smaller cross-sectional area results in a higher current flux density. Since the change in the SVF is larger for the real than for the virtual structures, the blue curve starts lower and ends higher than the red curve. The difference in current density at the current collector interface is also a result of a difference in the cross-sectional area: The real geometry has a roughly $5 \%$ larger contact area with the current collector than the virtual structure and therefore, in line with the previous argument, a smaller current flux density.

The analysis of the current densities in the two relevant phases indicates that the real and virtual structures exhibit quite identical characteristics. Therefore, we expect the virtual and real structures to behave similar regarding degradation and other local current flux dependent processes.

\subsection{Overpotential}

The distribution of the overpotential $\eta_{\text {So-El }}$, as defined by Eq. (5) and (6), is shown in Fig. 7(a) The overpotentials of real and virtual structures show a similar behavior. A positive overpotential indicates deintercalation/delithiation of lithium ions from the graphite into the electrolyte. It is clear that only deintercalation is taking place throughout the graphite electrode. The peaks at $0.00 \mathrm{~V}$ indicate the presence of interface areas between the solid phase and electrolyte pores, which are unconnected to the main pore space. This shows that the virtual microstructures contain more unconnected pore volume compared to the real structures, which is in agreement with Fig. 5(a) The second 


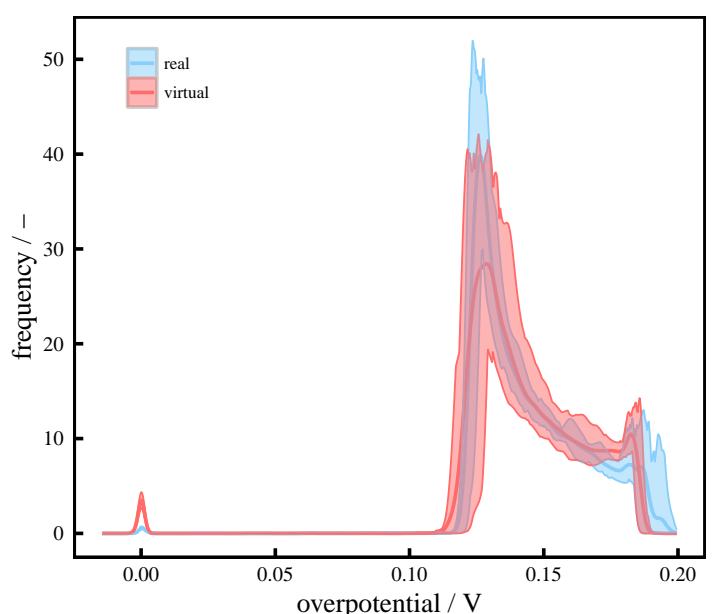

(a)

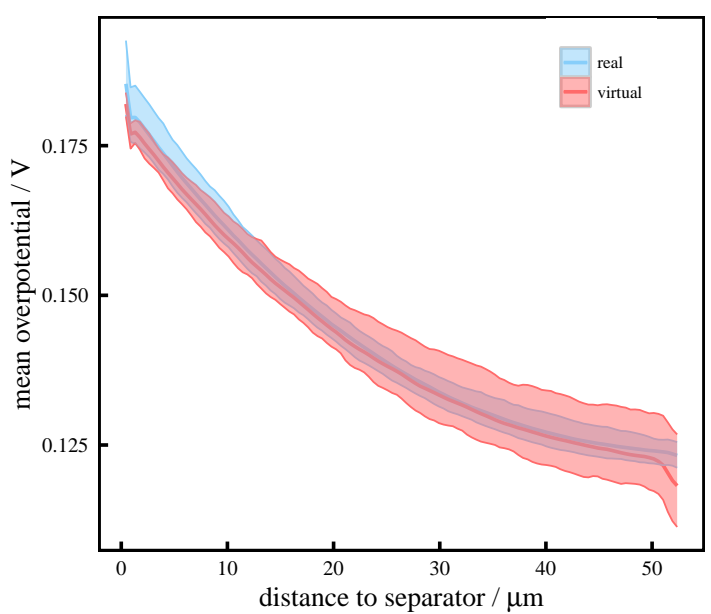

(b)

Figure 7: (a) Frequency distribution of the overpotential $\eta_{\text {So-El }}$ for the cut-outs of the virtual and real microstructures. A small peak at $0.0 \mathrm{~V}$, which indicates the presence of active surfaces at unconnected pore volumes, and a larger peak around $0.15 \mathrm{~V}$ exist. (b) Spatial distribution of the mean overpotential along the through-direction.

feature in the frequency plot consists of a large peak with a broad shoulder near higher overpotentials. The virtual structures exhibit a lower average feature compared to the real ones, whereas the real structures reach slightly higher overpotentials with a small shoulder. This additional feature is attributed to the differences in the solid volume fraction close to the separator (see Fig. 4).

The overpotential in the electrode as a function of distance in through-direction is shown in Fig. 7(b) The overpotential increases from the current collector towards the separator. This indicates an increase of the current flowing through the interface between solid and electrolyte. Due to the non-linear relation between the overpotential and the exchange current, the overpotential increase by about $50 \%$ leads to an increase of the average interface current of structures show a large spread for the most part and a kink close to the current collector. This kink is also attributed to the small morphological difference between virtual and real structures as described before (see e.g. Fig. 4). A larger overpotential close to the separator indicates a larger interface current. The spatial distribution of the exchange current density should exhibit a similar shape to the spatial distribution of the overpotential (see Fig. 7(b)), with the maximal

\subsection{Distribution of the lithium flux}

\subsubsection{Electrolyte phase}

The spatial distribution of the lithium ion flux inside the electrolyte, shown in Fig. 8(a), is identical for virtual and real structures. This, again, indicates that the two microstructure sources behave identical for the simulation of 
degradation and other processes.

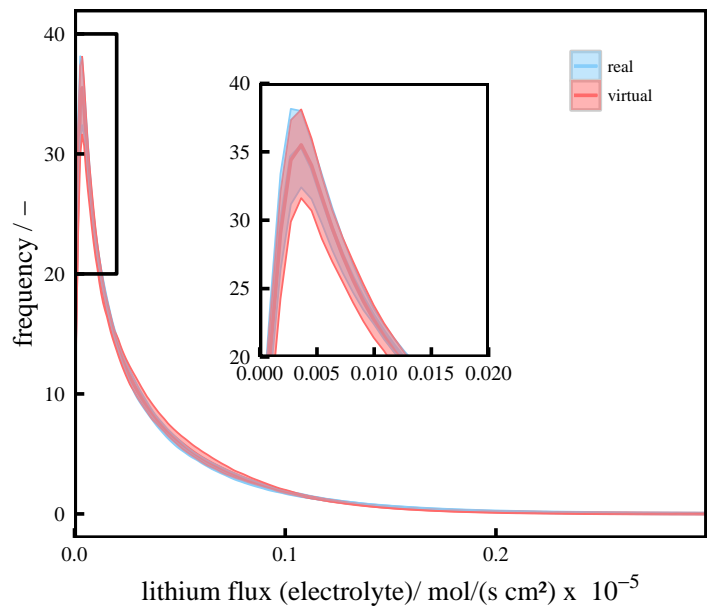

(a)

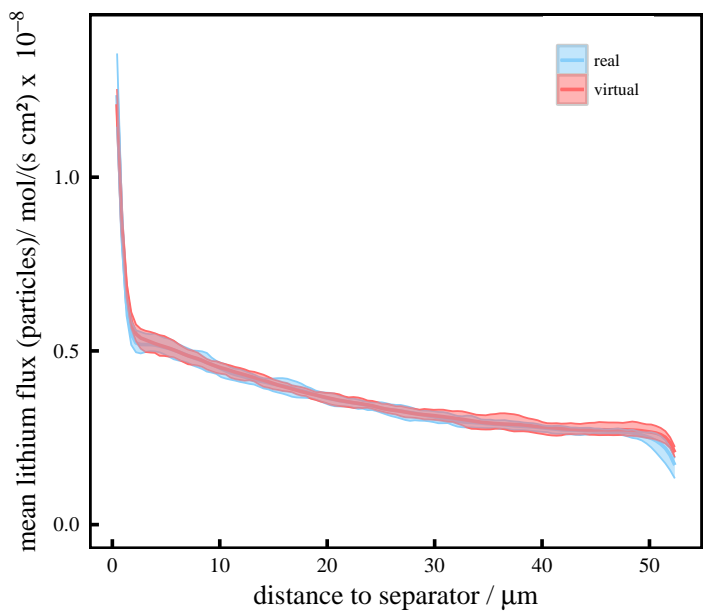

(b)

Figure 8: (a) Histogram of the lithium ion flux density inside the electrolyte for the high applied current. The lithium ion flux densities of the real and virtual structures show a nearly identical behavior. (b) The spatial distributions of the lithium flux inside the solid phase show quite good agreement. A small deviation can be seen close to the current collector. There, the real microstructures show a slightly lower lithium flux.

\subsubsection{Solid phase}

The spatial distribution of the lithium flux inside the solid phase is shown in Fig. 8(b) Since the solid electrode has a flat surface at the separator interface and therefore an increased active surface, that part of the electrode has an increased mean lithium flux density. There is also a deviation between the two geometry types in this region: The change in the lithium flux is more abrupt for the real than the virtual structures. On the other side (in throughdirection), the real structures show a smaller lithium flux. The reason of these two deviations is the difference in the solid volume fraction.

Apart from these features, the spatial lithium flux distributions inside the solid phase are quite identical for real and virtual structures. They both range over similar values and show a similar shape.

\section{Conclusion \& Outlook}

In this paper, we used spatially resolved electrochemical simulation in 3D to compare real (tomographically reconstructed) microstructures from lithium-ion cell anodes to the realizations of a stochastic microstructure model. The model was previously fitted to the tomographic image data and was validated using image characteristics from stochastic geometry. We used 20 cut-outs from real and simulated microstructures respectively. 
From the results of the electrochemical simulations we calculated several characteristics that were used for the comparison of both kinds of microstructures. Namely, the average cell potential, the spatial concentration of lithium in the electrolyte, the distribution of the current density inside the electrolyte and the solid phase, the distribution of the overpotential at the solid-electrolyte interface and the spatial distribution of the lithium flux inside the solid phase.

While a good overall fit of electrochemical characteristics computed for real and virtual microstructures could be shown, there are some small deviations that might slightly influence predictions made from simulation with virtual structures. The main difference is the inhomogeneous distribution of the volume fraction along through-direction, see Fig. 4. The increase of the volume fraction near the current collector and near the separator that is likely to be caused by the calendering [25, 26] during the production process is something that can be incorporated into the microstructure model by considering an inhomogeneous distribution of volume fraction in through-direction. Furthermore, there exist isolated pores in the anode, see Fig. 5(a) As there can be no ion exchange with the cathode this means that these pores do not take part in any reactions and thus reduce the active surface area as well as the active volume of the anode 27 28. To minimize the deviations between real and virtual structures a possible further extension of the microstructure model could be to prevent too many unconnected pores by considering a connectivity graph for the pores similar to the one for the particle connectivity, see [12]. The voxelized virtual structures also exhibit a slightly larger active surface area compared to the real microstructures. This results in a shift of the cell potential since a larger electrochemical active surface area leads to smaller overpotentials at the interfaces. This could be improved by extending our particle model and sampling procedure in a way that the expected surface area of the particles can be controlled like the expected volume of the particles. This would enable us to generate particles and entire microstructures where the active surface area can be adjusted.

If these three improvements are integrated into the stochastic microstructure model, a very good agreement between the virtual and real structures can be expected. Thus, such an extended microstructure model can be used to create structures that behave electrochemically very similar to the real ones. Additionally, it would be of great advantage to use the procedure described here for the fitting of the parameters in the stochastic microstructure model. The fitting procedure described in [12] could then be used for the initial values that are refined by the usage of electrochemical simulations. Using such a procedure microstructures with an even better accordance regarding electrochemical characteristics can be achieved.

Noteworthy is the fact that even though the real cut-outs are extracted from the same tomographic image, a variation in all electrochemical parameters is evident. It is therefore advisable to use several cut-outs or representations of real microstructures if one is interested in their performance.

Overall, as it is possible to generate virtual structures that behave similar to real structures extracted from tomographic 3D images, a next step could be to generate structures that correspond to more sophisticated structures that are not yet produced. This procedure is called virtual materials testing (VMT) and was already applied in related applications [7]. Our next goal is to utilize these techniques for the anode microstructure described in this paper. By this approach one is able to identify optimal structures with minimal experimental effort which can then later be 
produced and measured with the standard techniques. This is done by creating a large amount of (realistic) microstructures using systematic variations of parameters in the stochastic microstructure model. These virtual microstructures can be analyzed using electrochemical characteristics as described above and finally, optimal microstructures can be identified.

\section{Acknowledgements}

Financial support from the German Ministry of Education and Research (BMBF) in the framework Multibat 05M13PMA is gratefully acknowledged.

This research was supported in part by the bwHPC initiative and the bwHPC-C5 project ( funded by the Ministry of Science, Research and the Arts Baden-Württemberg (MWK) and the Germany Research Foundation (DFG) ) provided through associated computing services of the JUSTUS HPC facility at Ulm University.

365 [1] Y. Shin, A. Manthiram, J. Power Sources 126 (2004) 169-174.

[2] T. Waldmann, A. Iturrondobeitia, M. Kasper, N. Ghanbari, F. Aguesse, E. Bekaert, L. Daniel, S. Genies, I. J. Gordon, M. W. Löble, E. De Vito, M. Wohlfahrt-Mehrens, J. Electrochem. Soc. 163 (2016) A2149-A2164.

[3] H. Zhang, X. Yu, P. V. Braun, Nat. Nanotechnol. 6 (2011) $277-281$.

[4] Z. Wang, L. Zhou, X. W. Lou, Adv. Mater. 24 (2012) 1903-1911.

[5] T. Song, J. Xia, J.-H. Lee, D. H. Lee, M.-S. Kwon, J.-M. Choi, J. Wu, S. K. Doo, H. Chang, W. I. Park, D. S. Zang, H. Kim, Y. Huang, K.-C. Hwang, J. A. Rogers, U. Paik, Nano Lett. 10 (2010) 1710-1716.

[6] A. Vu, Y. Qian, A. Stein, Adv. Energy Mater. 2 (2012) 1056-1085.

[7] G. Gaiselmann, M. Neumann, V. Schmidt, O. Pecho, T. Hocker, L. Holzer, AIChE J. 60 (2014) 1983-1999.

[8] O. Stenzel, D. Westhoff, I. Manke, M. Kasper, D. P. Kroese, V. Schmidt, Model. Simul. Mater. Sci. Eng. 21 (2013) 055004.

[9] R. Thiedmann, O. Stenzel, A. Spettl, P. R. Shearing, S. J. Harris, N. P. Brandon, V. Schmidt, Comput. Mater. Sci. 50 (2011) $3365-3376$.

[10] D. Westhoff, J. J. van Franeker, T. Brereton, D. P. Kroese, R. A. J. Janssen, V. Schmidt, Model. Simul. Mater. Sci. Eng. 23 (2015) 045003.

[11] G. Gaiselmann, C. Tötzke, I. Manke, W. Lehnert, V. Schmidt, J. Power Sources 257 (2014) 52-64.

[12] J. Feinauer, T. Brereton, A. Spettl, M. Weber, I. Manke, V. Schmidt, Comput. Mater. Sci. 109 (2015) $137-146$.

[13] J. Newman, W. Tiedemann, AIChE J. 21 (1975) 25-41.

[14] R. Huggins, Advanced Batteries, Springer, New York, 2009.

[15] J. P. Meyers, M. Doyle, R. M. Darling, J. Newman, J. Electrochem. Soc. 147 (2000) 2930-2940.

[16] A. Latz, J. Zausch, J. Power Sources 196 (2011) 3296-3302.

[17] A. Latz, J. Zausch, Beilstein J. Nanotechnol. 6 (2015) 987-1007.

[18] J. Vetter, P. Novák, M. Wagner, C. Veit, K.-C. Möller, J. Besenhard, M. Winter, M. Wohlfahrt-Mehrens, C. Vogler, A. Hammouche, J. Power Sources 147 (2005) 269-281.

[19] T. Mitsch, Y. Krämer, J. Feinauer, G. Gaiselmann, H. Markötter, I. Manke, A. Hintennach, V. Schmidt, Materials 7 (2014) $4455-4472$.

[20] A. Latz, J. Zausch, Electrochim. Acta 110 (2013) 358 - 362.

[21] ITWM, BEST - Battery and Electrochemistry Simulation Tool, 2014. URL: http://itwm.fraunhofer.de/BEST

[22] G. B. Less, J. H. Seo, S. Han, A. M. Sastry, J. Zausch, A. Latz, S. Fell, J. Electrochem. Soc. 159 (2012) A697 - A704.

[23] J. Becker, A. Wiegmann, Math2Market GmbH, 2013. URL: http://www.geodict.com

[24] T. F. Fuller, M. Doyle, J. Newman, J. Electrochem. Soc. 141 (1994) 1-10.

[25] A. Väyrynen, J. Salminen, J. Chem. Thermodyn. 46 (2012) 80-85.

[26] H. Zheng, L. Tan, G. Liu, X. Song, V. S. Battaglia, J. Power Sources 208 (2012) 52-57.

[27] F. Sun, H. Markötter, D. Zhou, S. S. S. Alrwashdeh, A. Hilger, N. Kardjilov, I. Manke, J. Banhart, ChemSusChem 9 (2016) $946-950$.

[28] F. Sun, H. Markötter, K. Dong, I. Manke, A. Hilger, N. Kardjilov, J. Banhart, J. Power Sources 321 (2016) $174-184$. 
[29] M. Doyle, J. Newman, A. S. Gozdz, C. N. Schmutz, J.-M. Tarascon, J. Electrochem. Soc. 143 (1996) 1890-1903.

[30] L. O. Valoen, J. N. Reimers, J. Electrochem. Soc. 152 (2005) A882-A891.

[31] M. Morgan, Thin Solid Films 7 (1970) 313-323.

[32] J. Langer, V. Epp, P. Heitjans, F. A. Mautner, M. Wilkening, Phys. Rev. B - Condens. Matter Mater. Phys. 88 (2013) 1-9.

[33] M. Safari, C. Delacourt, J. Electrochem. Soc. 158 (2011) A562-A571.

[34] R. Chandrasekaran, T. F. Fuller, J. Electrochem. Soc. 158 (2011) A859-A871. 


\section{List of Tables}

$1 \quad$ Applied current densities with respect to the current collector area. ( $\mathrm{h}=$ high, $\mathrm{m}=$ medium, $\mathrm{l}=\mathrm{low})$. 21

$2 \quad$ Material and kinetic parameters used for the electrochemical simulations. . . . . . . . . . . . . 21

405 - 3 Statistics of surface area and solid volume for the real and virtual microstructures obtained from the voxelized datasets. . . . . . . . . . . . . . . . . . . . . . . . . . 21

4 Statistics of effective diffusion coefficient and effective conductivity for the real and virtual microstruc-

tures in throught direction obtained from the voxelized datasets. The thinner microstructures are the microstructures, which are reduced by 10 voxels on each side in through direction. . . . . . . . . . . 22 
Table 1: Applied current densities with respect to the current collector area. $(\mathrm{h}=$ high, $\mathrm{m}=$ medium, $1=$ low)

\begin{tabular}{ccc} 
name & value & C-rate \\
\hline \hline current h & $200 \mathrm{~mA} \mathrm{~m}^{-2}$ & $\approx 10 \mathrm{C}$ \\
current $\mathrm{m}$ & $20 \mathrm{~mA} \mathrm{~m}^{-2}$ & $\approx 1 \mathrm{C}$ \\
current $\mathrm{l}$ & $10 \mathrm{~mA} \mathrm{~m}^{-2}$ & $\approx \mathrm{C} / 2$ \\
\hline \hline
\end{tabular}

Table 2: Material and kinetic parameters used for the electrochemical simulations.

\begin{tabular}{|c|c|c|c|c|c|c|c|}
\hline \multirow[b]{2}{*}{ parameter/unit } & \multicolumn{2}{|c|}{ Graphite } & \multicolumn{2}{|c|}{ Li metal } & \multicolumn{3}{|c|}{ LiPF6 in PC/EC/DMC } \\
\hline & value & ref & value & ref & $\mathrm{p} / \mathrm{u}$ & value & ref \\
\hline$c_{\max } / \mathrm{kmol} \mathrm{m}^{-3}$ & 26.39 & [29] & & & $t_{+} /-$ & 0.399 & [30] \\
\hline$\sigma_{\mathrm{So}} / \mathrm{S} \mathrm{m}^{-1}$ & 1000 & {$[29,31,24]^{a}$} & & & $f_{ \pm} / \quad-$ & Eq. 6 in & [30] \\
\hline$D_{\mathrm{So}} / 10^{-13} \mathrm{~m}^{2} \mathrm{~s}^{-1}$ & 3.9 & [29, 32] & & & $\kappa_{\mathrm{El}} / \mathrm{S} \mathrm{m}^{-1}$ & Eq. 17 in & [30] \\
\hline$U_{0} / \mathrm{V}$ & $0.63+\ldots$ & 33] & & & $D_{\mathrm{El}} / \mathrm{m}^{2} \mathrm{~s}^{-1}$ & Eq. 14 in & 30] \\
\hline$i_{\mathrm{SoEl}}^{00} / \mathrm{A} \mathrm{m}^{5 / 2} \mathrm{~mol}^{-3 / 2}$ & $8.9 \cdot 10^{-7}$ & {$[24]^{b}$} & & & & & \\
\hline$i_{\mathrm{LiEl}}^{00} / \mathrm{A} \mathrm{mol}^{-1 / 2} \mathrm{~m}^{-1 / 2}$ & & & 0.364 & {$[34]^{a}$} & & & \\
\hline
\end{tabular}

${ }^{a}$ - Literature values vary, this value selected; ${ }^{b}$ - Calculated from literature for initial conditions;

Table 3: Statistics of surface area and solid volume for the real and virtual microstructures obtained from the voxelized datasets.

\begin{tabular}{ccccc}
\hline quantity & type & mean & std & unit \\
\hline \multirow{2}{*}{ surface area } & real & 7.72 & 0.34 & $\times 10^{-7} \mathrm{~m}^{2}$ \\
& vir & 7.80 & 0.40 & $\times 10^{-7} \mathrm{~m}^{2}$ \\
\hline \multirow{2}{*}{ solid volume } & real & 1.190 & 0.013 & $\times 10^{-12} \mathrm{~m}^{3}$ \\
& vir & 1.185 & 0.005 & $\times 10^{-12} \mathrm{~m}^{3}$ \\
\hline \multirow{2}{*}{ electrolyte volume } & real & 0.704 & 0.013 & $\times 10^{-12} \mathrm{~m}^{3}$ \\
& vir & 0.710 & 0.005 & $\times 10^{-12} \mathrm{~m}^{3}$ \\
\hline \hline
\end{tabular}


Table 4: Statistics of effective diffusion coefficient and effective conductivity for the real and virtual microstructures in throught direction obtained from the voxelized datasets. The thinner microstructures are the microstructures, which are reduced by 10 voxels on each side in through direction.

\begin{tabular}{ccccc}
\hline \hline quantity & type & \multicolumn{2}{c}{ mean (std) } \\
\hline & & \multicolumn{2}{c}{ complete } & thinner \\
microstructures
\end{tabular}


1 Schematic depiction of the stochastic model. (a) A random tessellation is generated, which roughly determines the particle shapes, sizes and locations. (b) A random graph describes how the particles connect. (c) The connected particles are then generated using Gaussian random fields on the sphere. (d) The auxilliary tools are deleted and the connected particle system is retained. (e) The particle system is morphologically smoothed to mimic the effect of binder on the contact region between particles. All pictures are taken from [12]. . . . . . . . . . . . . . . . . . . . 3

a 2 (a) The half-cell geometry used for the electrochemical simulations, here exemplified at the structure of a real cut-out. The different phases are (from top to bottom): Negative current collector, lithium 6- electrode, separator/electrolyte (transparent), porous graphite and positive current collector. (b) (main)

The cell potential of all full delithiation simulations show a good agreement between real and virtual microstructures. (inset) Comparison of the time when the cell potential reaches $1.2 \mathrm{~V}$, including the 5\%- and 95\%-quantiles. (c) (inset) The distribution of the electrolyte concentration for the short current pulse at the three different applied currents. (main) The $x$-values of the concentration distribution are rescaled in such a way that the peak at high concentration is at the same position for all currents and vice versa for the low concentration peak. This shows that the three curves describe the same behavior and can be converted into each other by simple scaling. $\ldots \ldots \ldots \ldots \ldots$

3 The average cell potential for real and virtual structures during the first $10 \mathrm{~s}$. The region between the $5 \%$ and $95 \%$-quantile is shown as shaded area. . . . . . . . . . . . . . . . 9

$4 \quad$ The average solid volume fraction along the through direction for the real and virtual structures. The

430 s. spatial distribution for the real cut-outs shows a region of more dense material close to the separator

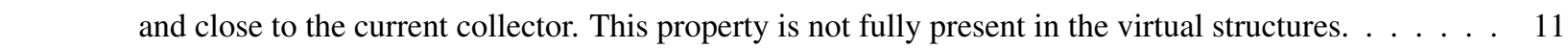

a 5 (a) The distribution of the electrolyte concentration for the electrode pore space and the separator (inset). The density is normed to unity area. The small peak at the initial concentration indicates unconnected pore volume. (b) The mean lithium concentration in the electrolyte as a function of the distance to the separator. The shape and height are nearly identical and both curves overlap in the region between the 5\% and 95\%-quantiles. (c) Spatial distribution of electrolyte concentration for two cut-outs: real (left) and virtual (right). Both have the same color scale (shown below). Larger particles can be seen in both structures. Also both cut-outs show electrolyte pores, which are less connected to the main pore space: (virtual) Orange part close to the blue and (real) dark red at the upper corner. 
$6 \quad$ Histogram of the current density inside the electrolyte (a) and the solid phase (b) for the high applied current after $10 \mathrm{~s}$. While lithium ions are the charge carrier in the electrolyte, electrons fulfill this role

in the solid phase. The distributions of the current densities in the real and virtual structures show a

nearly identical behavior. The spatial distributions of the average current density inside the electrolyte

445

(c) and inside the solid phase (d) for high applied current after $10 \mathrm{~s}$. The spatial distribution of the

average current densities of real and virtual structures differ inside the electrolyte close to the separator and inside the solid phase close to the current collector. . . . . . . . . . . . . . . . . . 13

7 (a) Frequency distribution of the overpotential $\eta_{\mathrm{So}-\mathrm{El}}$ for the cut-outs of the virtual and real microstruc-

tures. A small peak at $0.0 \mathrm{~V}$, which indicates the presence of active surfaces at unconnected pore vol-

450

umes, and a larger peak around $0.15 \mathrm{~V}$ exist. (b) Spatial distribution of the mean overpotential along the through-direction. . . . . . . . . . . . . . . . . . . . . . 15

a 8 (a) Histogram of the lithium ion flux density inside the electrolyte for the high applied current. The

lithium ion flux densities of the real and virtual structures show a nearly identical behavior. (b) The

spatial distributions of the lithium flux inside the solid phase show quite good agreement. A small

455 deviation can be seen close to the current collector. There, the real microstructures show a slightly

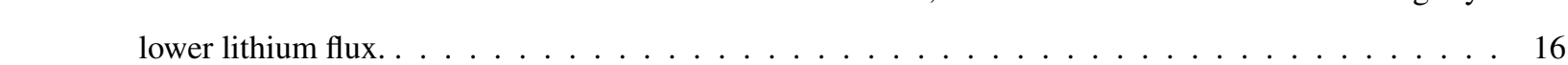

